\title{
AVALIAÇÃO ENERGÉTICA E SIMULAÇÃO DE SISTEMAS DE EVAPORAÇÃO COM RECOMPRESSÃO DE VAPOR EM UMA INDÚSTRIA DE LEITE
}

\author{
A. J. P. BEUREN ${ }^{1 *}$, A. L. CATTO ${ }^{1}$ \\ ${ }^{1}$ Centro Universitário Univates, Faculdade de Engenharia Química \\ E-mail para contato: augustobeuren@ hotmail.com
}

\begin{abstract}
RESUMO - Este estudo de caso foca na aplicação dos conhecimentos em operações unitárias, balanços de massa e energia para quantificar potenciais economias de energia em um sistema de evaporação com 5 efeitos de uma indústria de laticínios no Rio Grande do Sul. Evaporadores modernos de leite utilizam tipicamente tanto a tecnologia de recompressão térmica quanto mecânica de vapor como meio para atingir uma alta eficiência de energia. Uma mudança significativa na eficiência energética é atingida neste estudo ao apresentar um novo sistema de evaporação de duplo efeito com recompressão de vapor. O novo sistema requerendo $21,6 \%$ menos vapor ao custo de $130 \mathrm{kWh}$ energia elétrica. Este sistema ainda reduziria as emissões em 1291,14 t $\mathrm{CO}_{2}$-e/ano.
\end{abstract}

\section{INTRODUÇÃO}

A indústria de alimentos é, em maior parte, a maior consumidora de água e energia, tendo a responsabilidade de garantir que ambos recursos sejam empregados de forma moderada e eficiente (Klemes et al. 2008).

Nos sistemas de evaporação, o objetivo principal é concentrar uma solução contendo um soluto não volátil e uma solução volátil. Na grande maioria das operações de evaporação, o solvente é a água e este processo consiste em evaporar uma porção do solvente até se obter um produto concentrado em soluto (Mccabe et al. 2005; Geankoplis, 1993). No caso específico do processamento do leite em pó na indústria, o processo de evaporação a vácuo remove a primeira parcela de água e transforma o líquido fino em um concentrado de leite com alta viscosidade (Piscecký, 2012). A evaporação de múltiplo efeito é o principal meio em uso para economia no consumo de energia, sendo que a economia de vapor aumenta com o aumento do número de efeitos (Perry e Green, 2008).

A maneira mais simples de reduzir o gasto de energia com evaporação é comprimir o vapor em um evaporador de simples efeito para o vapor ser utilizado como meio aquecedor no mesmo evaporador. A compressão pode ser realizada por meio mecânico (MVR do inglês Mechanical Vapour Recompression) ou por jatos de vapor (do inglês Thermal Vapour Recompression) (Perry e Green, 2008). Das duas técnicas, o MVR requer investimento mais alto de capital, mas pode reduzir muito o número de efeitos e assim, diminuir o uso global de energia e custos de operação (Hanneman e Robertson, 2005). 
O foco deste estudo de caso de uma indústria de processamento de leite é avaliar o atual sistema de evaporação de 5 efeitos com um compressor TVR, propondo um novo sistema de evaporação com somente dois efeitos e dois compressores TVR e um MVR que minimizariam o consumo total de energia.

\section{METODOLOGIA}

Uma combinação das operações unitárias, modelagem e simulação de processo, formam a base para o desenvolvimento de um novo sistema de evaporação e otimização do processo. As operações unitárias e a modelagem do processo ajudam a determinar os múltiplos efeitos de se realizar uma mudança no processo.

\subsection{Descrição do Modelo de Processo}

Vários balanços detalhados de calor e massa com diferentes configurações de um sistema de evaporadores de filme descendente (falling film) com múltiplos efeitos incluindo recompressão de vapor foram implementados em planilhas do MS Excel. O modelo foi testado e validado pela atual configuração utilizada na indústria do estudo de caso e aplica propriedades padrões de vapor de água (IAPWS IF-97) e propriedades do leite (MAF, 1996).

O objetivo de diferentes configurações é minimizar o consumo de energia. A eficiência de um evaporador pode ser expressa em economia de vapor, conforme a Equação 1, onde $m_{\text {evap }}$ é a massa de água evaporada e $m_{\text {vapor }}$ é a massa de vapor condensada.

$$
\text { Economia de vapor }=\frac{\dot{m}_{\text {evap }}}{\dot{m}_{\text {vapor }}}
$$

A pressão de vapor de soluções aquosas é menor que a da água para uma mesma temperatura. Consequentemente, para uma determinada pressão, o ponto de ebulição da solução é maior que da água. Este aumento no ponto de ebulição é conhecido como BPE (boiling point elevation) e pode ser calculado conforme as Equações 2 e 3, onde $R$ é a constante dos gases ideais, $T_{W B}$ temperatura de ebulição da água e $x_{w}$ a fração molar de água.

$$
\begin{aligned}
B P E & =\frac{-R T_{w b}^{2} \ln x_{w}}{\Delta h_{V}} \\
\Delta h_{V} & =57,222-44,3 T_{w b} \mathrm{~J} \mathrm{~mol}^{-1}
\end{aligned}
$$

O balanço de massa (Equação 4) relaciona todas as correntes que entram e saem de cada efeito, porém a massa de vapor que entra é igual ao condensado, podendo este ser desprezado. A Equação 5 apresenta um balanço de massa por componente, onde $w$ é a fração de sólidos.

$$
\begin{aligned}
& \dot{m}_{\text {evaporada }}=\dot{m}_{\text {alimentação }}-\dot{m}_{\text {concentrado }} \\
& \dot{m}_{\text {alimentação }} w_{\text {alimentação }}=\dot{m}_{\text {conconcentrado }} w_{\text {conconcentrado }}
\end{aligned}
$$


Para o balanço de energia, assume-se que não há vazamentos ou infiltrações, que o fluxo de gases não condensáveis é negligenciado e que as perdas de calor do evaporador são desprezíveis. Considera-se que a diferença entre a entalpia do vapor e seu condensado é simplesmente $\lambda_{\text {vapor }}$, que é o calor latente de condensação do vapor. Na ausência de perdas térmicas, o calor transferido aos tubos é igual ao calor transferido ao licor (Equação 6), onde $H_{\text {evap }}$ é a entalpia de vapor saturado e $H_{\text {alim }}$ e $H_{\text {conc }}$ são entalpias de líquido saturado.

$$
q=\dot{m}_{\text {vapor }} \lambda_{\text {vapor }}=\left(\dot{m}_{\text {alim. }}-\dot{m}_{\text {conc }}\right) H_{\text {evaporado }}-\dot{m}_{\text {alim. }} H_{\text {alim. }}+\dot{m}_{\text {conc }} H_{\text {conc. }}
$$

O reciclo de calor ou simplesmente razão de arraste $\mu$ de um compressor TVR no sistema de evaporação pode ser determinada pela Equação 7 e seu valor depende da pressão de vapor vivo, $P_{S}$, vapor fornecido, $P_{V}$, e a descarga de vapor $P_{D}$, conforme a Equação 8.

$$
\begin{aligned}
& \mu=\frac{\text { massa de vapor recirculado }}{\text { massa de vapor atuante }} \\
& \mu=R_{S}=0,40 e^{4,6 \ln \left(\frac{P_{d}}{P_{V}}\right) / \ln \left(\frac{P_{S}}{P_{V}}\right)}
\end{aligned}
$$

O aumento de pressão que um compressor MVR pode proporcionar pode ser calculado pela Equação 9, onde $\Delta P$ é a razão da pressão de saída pela pressão de entrada, $\psi$ é o coeficiente de pressão, $u_{\text {tip }}$ é a velocidade do ventilador, $M$ é a massa molar de água, $R$ constante dos gases e $T$ é o aumento da temperatura. A energia necessária para este compressor pode ser determinada pela Equação 10, sendo sua eficiência isentrópica típica de $\eta=80 \%$, onde $Q$ é a taxa média de fluxo volumétrico calculado pelas densidades de entrada e saída. $\Delta h_{f a n}$ é a mudança na entalpia do vapor ao passar pelo ventilador.

$$
\begin{aligned}
& \Delta P=\frac{P_{\text {oUT }}}{P_{I N}}=1+\frac{\psi u_{t i p}^{2} M}{2 R T} \\
& P_{O}=\frac{Q \Delta P}{\eta}=\dot{m}_{\text {evap }} \Delta h_{f a n}
\end{aligned}
$$

\section{ESTUDO DE CASO DE UM SISTEMA DE EVAPORAÇÃO DE LEITE}

\subsection{Descrição do processo e parâmetros de processamento}

Os sistemas de evaporação de leite possuem diversos processos que ocorrem em sua redondeza que proporcionam oportunidades para integração de calor. A integração direta entre o sistema de evaporação e o processo de separação de leite não é permitida, sendo assim, ao invés de integrar através de todo o sistema, a integração local faz-se necessária.

O processo de tratamento térmico do leite é uma combinação de temperatura e tempo, no qual afeta o grau de desnaturação do leite (Oldfield et al., 2005) e o sabor do leite em pó (Baldwind et al., 1991). O sistema de evaporação vem em seguida ao processo de tratamento térmico. $\mathrm{O}$ evaporador de filme descendente com vários efeitos concentra o leite padronizado de $12 \%$ para $48 \%$ de sólidos. Normalmente os evaporadores operam em uma faixa de temperatura de $45-73^{\circ} \mathrm{C}$ para evitar a desnaturação das proteínas. O último passo do 
processamento é a secagem por Spray Dryer. Após deixar o evaporador, o leite é homogeneizado, atomizado e seco com ar quente para formar leite em pó.

\subsection{Design do sistema de evaporação e dados das correntes}

Este estudo de caso conta com um sistema de evaporação de 5 efeitos com compressor TVR (Figura 1). O primeiro efeito é integrado com um TVR que utiliza parte do vapor que deixa o segundo efeito. Este sistema de evaporação apresentou uma economia de 5,558, ou seja, com $1 \mathrm{~kg}$ de vapor é possível evaporar $5,558 \mathrm{~kg}$ de água.

Figura 1 - Diagrama do evaporador com múltiplos efeitos e recompressão de vapor.

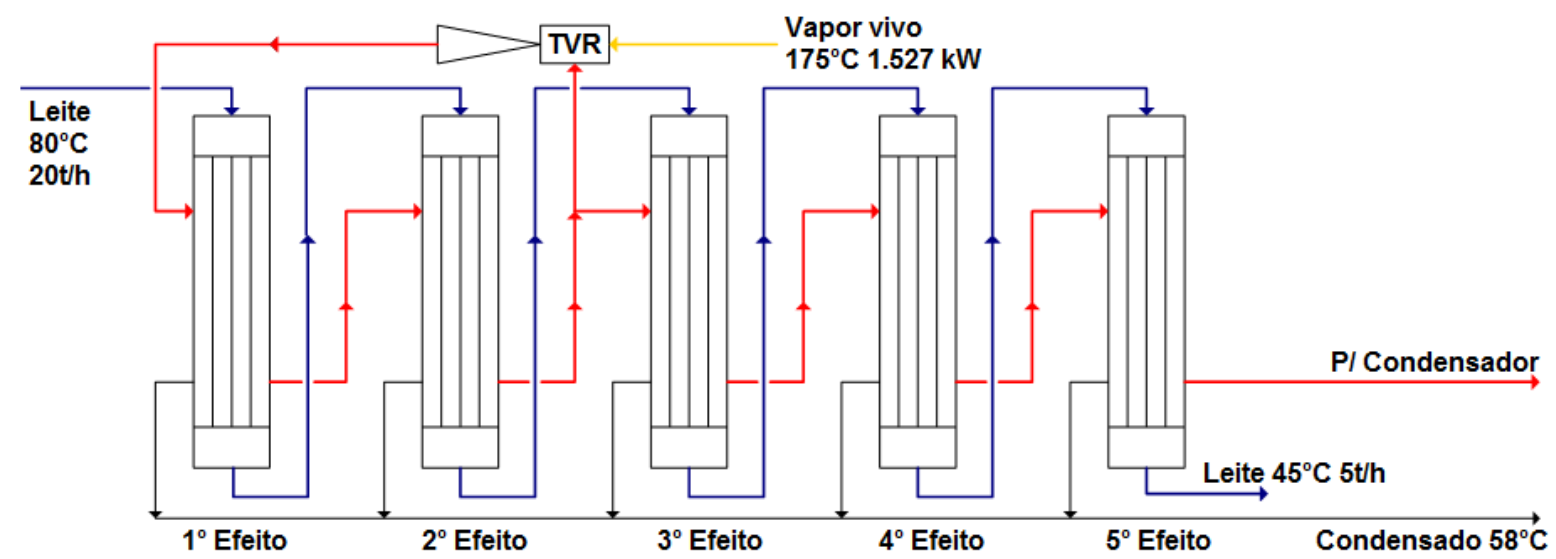

\section{RESULTADOS E DISCUSSÕES}

Após simulações em planilhas do MS Excel, as informações de economia de vapor, consumo de vapor, consumo de energia elétrica e configuração do sistema são apresentadas na Tabela 1. Pode-se perceber que com menos efeitos, integrados com a recompressão de vapor, é possível atingir um nível superior de economia de vapor ao custo de uma pequena elevação no consumo de energia elétrica. Este novo sistema de evaporação de duplo efeito com recompressão de vapor está apresentado na Figura 2.

Tabela 1 - Resumo das configurações e respectivos dados.

\begin{tabular}{cccc}
\hline Configuração & Economia de vapor & Consumo de vapor & Consumo elétrico \\
\hline Simples efeito & 0,936 & $9113,3 \mathrm{~kW}$ & - \\
\hline 5 efeitos & 4,490 & $1900 \mathrm{~kW}$ & - \\
\hline 5 efeitos com TVR & 5,588 & $1527 \mathrm{~kW}$ & - \\
\hline 2 efeitos c/ 2 TVR+MVR & 9,284 & $919 \mathrm{~kW}$ & $130 \mathrm{~kW}$ \\
\hline
\end{tabular}

A Figura 2 apresenta o novo sistema de evaporação mais econômico que o atual sistema de 5 efeitos com um compressor TVR. Outros estudos como os de Liew et al. (2014), Walmsley (2016), Walmsley et al. (2016) também sugerem uma maior utilização de recompressores de vapor nos sistemas de evaporação. Esta proposta de otimização do processo de evaporação gera grande alteração no design atual, porém, como a indústria tem planejamento de expansão da sua produção, este sistema poderia ser instalado operando 
paralelamente ao sistema antigo, a fim de aumentar a produção e obter ganhos energéticos e econômicos ao processo.

Figura 2 - Fluxograma do novo sistema de evaporação.

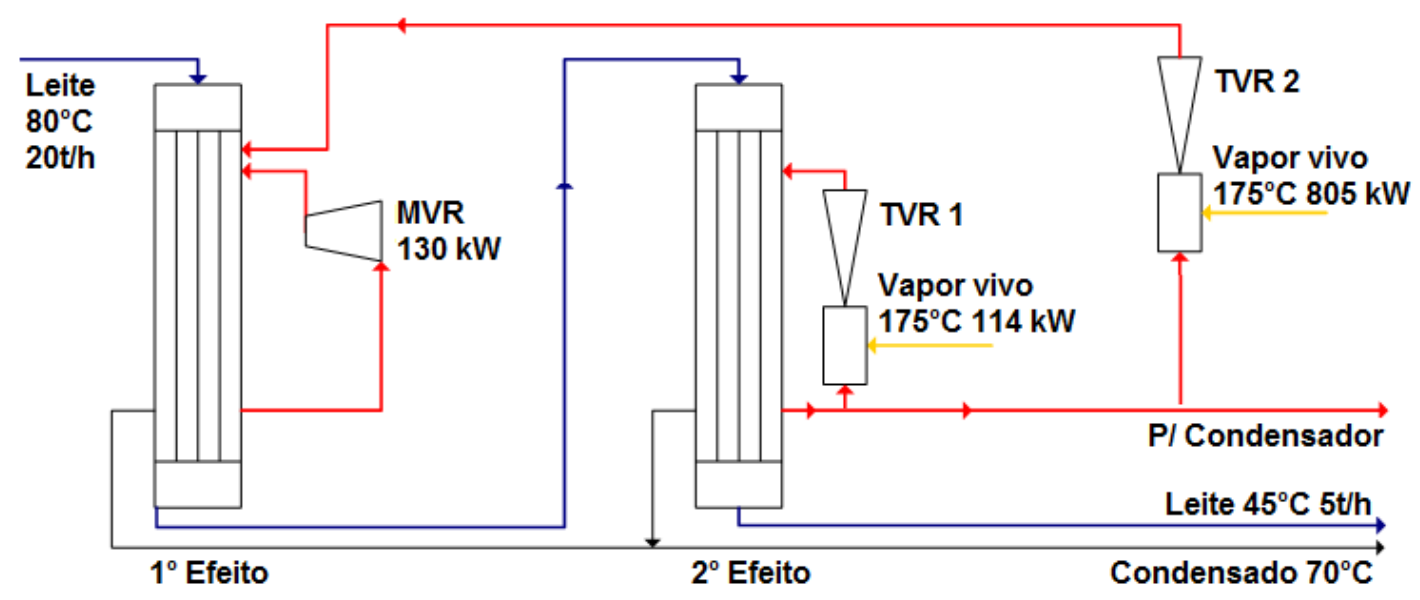

Na redução das emissões para implementação do novo sistema também foi levada em consideração os fatores de emissão da energia elétrica $\left(0,129\right.$ t $\mathrm{CO}_{2}$-e/MWh), valor que é praticamente a metade do fator de emissão do carvão $\left(0,270\right.$ t $\mathrm{CO}_{2}$-e/MWh) (Walmsley, 2014). A Tabela 2 apresenta um levantamento de emissões e a economia de vapor relacionando o atual e o novo sistema proposto. Será possível reduzir as emissões em 1291,14 $\mathrm{t} \mathrm{CO}_{2}$ por ano.

Tabela 2 - Redução na emissão de $\mathrm{CO}_{2}$ entre os diferentes sistemas de evaporação

\begin{tabular}{cccc}
\hline Configuração & $\begin{array}{c}\text { Consumo de vapor } \\
{[\mathbf{k W}]}\end{array}$ & $\begin{array}{c}\text { Consumo elétrico } \\
{[\mathbf{k W}]}\end{array}$ & $\begin{array}{c}\text { Redução de emissões } \\
{[\mathbf{t} \text { CO2-e/ano }]}\end{array}$ \\
\hline 5 efeitos com TVR & 1527 & - & - \\
\hline $\begin{array}{c}\text { Duplo efeito com } \\
\text { 2 TVR + 1 MVR }\end{array}$ & 919 & 130 & 1291,14 \\
\hline
\end{tabular}

Conforme Walsmley et al. (2016), pela aplicação de conceitos e métodos de integração de energia como o Pinch Analysis (PA) na planta industrial, é possível reduzir ainda mais as emissões de $\mathrm{CO}_{2}$, bem como custos de energia elétrica e térmica. $\mathrm{O}$ mesmo estudo também aprimora ainda mais o design deste sistema de evaporação de duplo efeito com recompressão de vapor ao utilizar somente compressores do tipo MVR, mas é importante observar a relação do custo da energia elétrica e térmica.

\section{CONCLUSÃO}

O novo sistema de evaporação com duplo efeito e recompressão de vapor mostra-se mais eficiente na utilização do vapor vivo, representando uma redução de $21,6 \%$, sendo assim uma oportunidade da indústria para economizar recursos e que pode ser implantada em caso de expansão da produção. Esta alteração proporciona uma redução significativa no número de efeitos do sistema de evaporação, o que acarretaria um menor custo de implantação e redução estimada das emissões de $\mathrm{CO}_{2}$ em 1291,14 t $\mathrm{CO}_{2}$-e/ano. 


\section{REFERÊNCIAS}

BALDWIN, A. J.; COOPER, H. R.; Effect of preheating treatment and storage on the proprieties of whole powder. Changes in sensory proprieties. Neth Milk Dairy J. V. 45, 97-116, 1991.

GEANKOPLIS, C. J. Transport Processes and Unit Operations. 3. ed. New Jersey: Prentice Hall PTR, 1993.

HANNEMAN, H.; ROBERTSON, L. J. Heat Recovery Systems, in: Energy Use Dairy Process. International Dairy Federation, Brussles, Belgium, 2005.

KLEMES, J. J.; SMITH, R.; KIM, J. K. Handbook of water and energy management in food processing. Cambridge, UK: Woodhead Publishing; 2008.

LIEW, P. Y.; WAN A. S. R.; LIM, J. S. VARBANOV. P. S.; KLEMES, J. J.; ABDUL M. Z. Total Site Heat Integration incorporating the water sensible heat. J. Clean. Prod. v. 77, 94-104, 2014.

MAF Quality Management. Physical properties of dairy products. 3rd ed. Hamilton, New Zealand: MAF Quality Management, 1996.

MCCABE, W. L.; SMITH, J. C.; HARRIOTT, P. Unit Operations of Chemical Engineering. 7. ed. Boston: McGraw-Hill, c2005.

OLDFIELD D. J.; TAYLOR, M. W.; SINGH H. Effect of preheating and other process parameters on whey protein reactions during skim milk powder manufacture. Int. Dairy J. V. 11. 15-501, 2005.

PERRY, R. H.; GREEN, D. W. (Eds). Perry's Chemical Engineers' Handbook. 8. ed. New York: McGraw-Hill, 2008.

PISCECKÝ, J. Handbook of Milk Powder Manufacture. 2 ed. Copenhagen, Denmark: GEA Niro, 2012.

WALMSLEY, M. R. W.; WALMSLEY, T. G.; ATKINS, M. J.; KAMP, P. J. J.; NEALE, J. R. Minimising carbon emissions and energy expended for electricity generation in New Zealand through to 2050. Appl. Energy. V. 65, 135-656, 2014.

WALMSLEY, T. G. A Total Site Heat Integration design method for integrated evaporation systems including vapour recompression. J. of Cleaner Production, 2016.

WALMSLEY, T. G.; ATKINS, M. J.; WALMSLEY, M. R. W.; NEALE, J. R. Appropriate placement of vapour recompression in ultra-low energy industrial milk evaporation systems using Pinch Analysis, Energy 2016. 